\title{
ON THE COMPLETENESS OF THE SPACE OF COMPACT SUBSETS
}

\author{
PHILLIP ZENOR
}

\begin{abstract}
The purpose of this paper is to show that if $X$ is a completely regular $T_{1}$-space, then $\mathfrak{C}(X)$, the space of all compact subsets of $X$ with the Vietoris topology, is realcompact (topologically complete in the sense of Dieudonne) if and only if $X$ is realcompact (topologically complete in the sense of Dieudonné).
\end{abstract}

Following the convention of [1], if $\left\{U_{1}, U_{2}, \cdots, U_{n}\right\}$ is a collection of open sets, then $\left\langle U_{1}, U_{2}, \cdots, U_{n}\right\rangle$ will denote the subset of $\mathfrak{e}(X)$ to which the compact set $H$ belongs if and only if (i) $H \subset \bigcup_{i=1}^{n} U_{i}$ and (ii) $H \cap U_{i} \neq \varnothing$ for $i=1,2, \cdots, n$. The sets of the form $\left\langle U_{1}, U_{2}, \cdots, U_{n}\right\rangle$ are the basic open sets for the Vietoris topology on $\mathfrak{e}(X)$. Note that on $\mathfrak{e}(X)$ the Vietoris topology is the same as the topology induced by the Hausdorff metric providing that $X$ is metric [1, Theorem 3.3].

Recall that the completely regular space $X$ is realcompact if and only if $X$ is homeomorphic to a closed subset of a topological product of copies of the reals and $X$ is Dieudonné complete if $X$ is homeomorphic to a closed subspace of a product of metric spaces.

Notation. If $f$ is a continuous function from $X$ into $Y$, then $\tilde{f}$ will denote the function from $\mathfrak{e}(X)$ into $\mathfrak{e}(Y)$ defined by $\tilde{f}(H)$ $=\{f(x) \mid x \in H\}$.

Lemma A. If $f$ is a continuous function from $X$ into $Y$, then $\tilde{f}$ is continuous.

Proof. Let $\left\langle U_{1}, U_{2}, \cdots, U_{n}\right\rangle$ be a basic open set in $\mathcal{C}(Y)$ that contains $\tilde{f}(H)$. Then $\left\langle f^{-1}\left(U_{1}\right), \cdots, f^{-1}\left(U_{n}\right)\right\rangle$ is a basic open set in $\mathcal{e}(X)$ containing $H$ such that if $K$ is in $\left\langle f^{-1}\left(U_{1}\right), \cdots, f^{-1}\left(U_{n}\right)\right\rangle$, then $\tilde{f}(K)$ is in $\left\langle U_{1}, \cdots, U_{n}\right\rangle$.

LemMA B. Suppose that $\left\{X_{a}, f_{b}^{a}\right\}$ is an inverse system over the directed set $A$. Then $\mathrm{e}\left(\mathrm{inv} \lim \left\{X_{a}, f_{b}^{a}\right\}\right)$ is homeomorphic to inv $\lim \left\{\mathfrak{e}\left(X_{a}\right), \tilde{f}_{b}^{a}\right\}$.

Proof. For each $a$ in $A$, let $P_{a}$ denote the projection of inv $\lim \left\{X_{a}, f_{b}^{a}\right\}$ into the coordinate space $X_{a}$ and let $P_{a}^{*}$ denote the

Received by the editors October 27, 1969.

AMS 1969 subject classifications. Primary 5425, 5452.

Key words and phrases. Hyperspace, realcompactness, completeness, inverse limits. 
projection of inv $\lim \left\{\mathfrak{e}\left(X_{a}\right), \tilde{f}_{b}^{a}\right\}$ into $\mathfrak{C}\left(X_{a}\right)$. For each element $H$ in $\mathcal{C}\left(\right.$ inv $\left.\lim \left\{X_{a}, f_{b}^{a}\right\}\right)$, let $T(H)=\left\{P_{a}(H)\right\}_{a \in A}$. Since if $a<b$ are elements of $A$, then $P_{a}=f_{a}^{b} \cdot P_{b}$, it follows that $T(H)$ is a point in inv $\lim \left\{\mathfrak{e}\left(X_{a}\right), \tilde{f}_{b}^{a}\right\}$. Thus, $T$ is a function from $\mathfrak{e}\left(\right.$ inv $\left.\lim \left\{X_{a}, f_{b}^{a}\right\}\right)$ into inv $\lim \left\{\mathfrak{e}\left(X_{a}\right), \tilde{f}_{b}^{a}\right\}$. It is obvious that $T$ is one-to-one and onto.

To see that $T$ is continuous, we will show that for each $a^{\prime}$ in $A, P_{a^{\prime}}^{*} \cdot T$ is continuous. Let $\left\langle U_{1}, \cdots, U_{n}\right\rangle$ be a basic open set in $\mathfrak{e}\left(X_{a^{\prime}}\right)$ containing $H_{a^{\prime}}=P_{a^{\prime}}^{*} \cdot T(H)$. It is easy to see that $\left\langle P_{a^{\prime}}^{-1}\left(U_{1}\right), \cdots, P_{a^{\prime}}^{-1}\left(U_{n}\right)\right\rangle$ is an open set in $\mathcal{C}\left(\right.$ inv $\left.\lim \left\{X_{a}, f_{b}^{a}\right\}\right)$ containing $H$ such that if $K$ is in $\left\langle P_{a^{\prime}}^{-1}\left(U_{1}\right), \cdots, P_{a^{\prime}}^{-1}\left(U_{n}\right)\right\rangle$, then $P_{a^{\prime}}^{*}(T(K))$ is in $\left\langle U_{1}, U_{2}, \cdots, U_{n}\right\rangle$.

Finally, to see that $T$ is open, let $\left\{U_{1}, \cdots, U_{n}\right\}$ denote a finite collection of open sets in inv $\lim \left\{X_{a}, f_{b}^{a}\right\}$ such that $\left\langle U_{1}, \cdots, U_{n}\right\rangle$ contains the element $H$ of $\mathfrak{e}\left(\operatorname{inv} \lim \left\{X_{a}, f_{b}^{a}\right\}\right)$. For each $i=1,2, \cdots, n$, (a) let $x_{i}$ denote a point of $H$ in $U_{i}$ and (b) let $a_{i}$ denote an element of $A$ and $U_{i}^{\prime}$ an open set in $X_{a_{i}}$ such that $x_{i} \in P_{a_{i}}^{-1}\left(U_{i}^{\prime}\right) \subset U_{i}$. Let $\left\{b_{1}, b_{2}, \cdots, b_{j}\right\}$ denote a subset of $A$ and let $\left\{V_{1}, V_{2}, \cdots, V_{j}\right\}$ denote a collection of sets such that (a) for $i=1,2, \cdots, j, V_{i}$ is open in $X_{b_{i}}$ and contains a point of $P_{b_{i}}(H)$ and (b) $\left\{P_{b_{1}}^{-1}\left(V_{1}\right), \cdots, P_{b_{j}}^{-1}\left(V_{j}\right)\right\}$ covers $H$ and refines $\left\{U_{1}, \cdots, U_{n}\right\}$. Let $c$ denote an upperbound in $A$ for $\left\{a_{1}, a_{2}, \cdots, a_{n}, b_{1}, \cdots, b_{j}\right\}$. Then

$\operatorname{inv} P_{c}^{*}\left(\left\langle\operatorname{inv} f_{a_{1}}^{c}\left(U_{1}^{\prime}\right), \operatorname{inv} f_{a_{2}}^{c}\left(U_{2}^{\prime}\right), \cdots, \operatorname{inv} f_{a_{n}}^{c}\left(U_{n}^{\prime}\right)\right.\right.$,

$$
\left.\left.\operatorname{inv} f_{b_{1}}^{c}\left(V_{1}\right), \cdots, \operatorname{inv} f_{b_{j}}^{c}\left(V_{j}\right)\right\rangle\right)
$$

is an open set in inv $\lim \left\{\mathfrak{e}\left(X_{a}\right), \tilde{f}_{b}^{a}\right\}$ that contains $T(H)$ and is a subset of $T\left(\left\langle U_{1}, U_{2}, \cdots, U_{n}\right\rangle\right){ }^{1}$

THEOREM. If $X$ is a completely regular $T_{1}$-space, then:

1. $\mathfrak{e}(X)$ is realcompact if and only if $X$ is realcompact.

2. $\mathfrak{e}(X)$ is Dieudonné complete if and only if $X$ is Dieudonné complete.

Proof. $X$ is homeomorphic to the closed subspace of $\mathfrak{e}(X)$ consisting of all the degenerate subsets of $X$. Thus if $\mathfrak{e}(X)$ is complete (realcompact), then $X$ is complete (realcompact).

Suppose that $X$ is realcompact. Then, by [2, Theorem 2], $X$ is homeomorphic to an inverse limit of an inverse system of separable metric spaces over a directed set. Now, if $M$ is a separable metric space, $\mathfrak{e}(M)$ has a countable basis; thus, if $M$ is a separable metric space, $\mathfrak{C}(M)$ is a separable metric space. It now follows from Lemma $\mathrm{B}$ that $\mathfrak{e}(X)$ is homeomorphic to an inverse limit of an inverse system

1 inv $f=f^{-1}$. 
of separable metric spaces over a directed set. According to [2, Theorem 2], $\mathfrak{e}(X)$ is realcompact.

If $X$ is Dieudonné complete, then by [3, Theorem 3.1], $X$ is homeomorphic to the inverse limit of an inverse system of metric spaces. By Lemma $\mathrm{B}, \mathfrak{e}(X)$ is homeomorphic to the inverse limit of an inverse system of metric spaces over a directed set; thus $\mathcal{e}(X)$ is homeomorphic to a closed subset of a product of metric spaces, which completes the proof.

The referee has pointed out to the author that the theorem is true if "completeness in the sense of Dieudonné" is replaced by "completeness in the sense of Cech." An argument, as outlined by the referee, can be obtained by noting that a space is Cech complete if and only if $X$ is the inverse limit of an inverse system of locally compact spaces over a countable directed set. This result now follows from Lemma B and [1, Theorem 4.9.12].

\section{REFERENCES}

1. E. Michael, Topologies on spaces of subsets, Trans. Amer. Math. Soc. 71 (1951), 152-182. MR 13, 54.

2. P. Zenor, Extending completely regular spaces with inverse limits, Glasnik Mat. Ser III 5 (1970).

3. ——, Extensions of topological spaces, (to appear).

Auburn University, Auburn, Alabama 36830 